\title{
Initial Anticoagulant Management of Deep Vein Thrombosis/Venous Thromboembolism in Primary Care: Review of Current Approaches
}

\author{
Authors: \\ *Pablo Millares Martin \\ Whitehall Surgery, Wortley Beck Health Centre, Leeds, UK \\ ${ }^{*}$ Correspondence to Pablo.martin@nhs.net \\ Disclosure: The author has declared no conflicts of interest. \\ Received: $\quad 18.04 .20$ \\ Accepted: $\quad 13.05 .20$ \\ Keywords: $\quad$ Deep vein thrombosis (DVT), managed care, patient-centred medical home, \\ pharmacology, primary care, procedures, venous thromboembolism (VTE). \\ Citation: $\quad$ EMJ Hematol. 2020;8[1]:93-99
}

\begin{abstract}
Background and Aims: The initial management of deep vein thrombosis is starting to happen in general practice. New treatments are available to allow this shift, but guidance is variable. The aim of this study was to understand current choices used in general practice in the UK and to determine if there is a more efficient treatment, considering variability observed locally.
\end{abstract}

Methods: A systematic literature review and freedom of information requests to England's 198 clinical commissioning groups (CCG) were used to gather information on treatment options and current uses, respectively. Over 100 papers were assessed, and information from 177 CCG was obtained.

Results: There is noninferiority between injectable low-molecular-weight heparin and novel oral anticoagulant treatments. Fifteen CCG offered variable, but also limited, options of treatment. Patient choice was not necessarily considered.

Conclusion: There is variability in England on availability and choice of therapy for the initial management of deep vein thrombosis at present, which may also be the case elsewhere. The implementation of evidence-based guidelines should be carefully considered in all settings and countries.

\section{INTRODUCTION}

Healthcare continuously evolves, which requires the reassessment of management pathways based on new drugs becoming available and on clinical factors. When considering new medications, the costs and the efficiency are the main influences. Clinical factors include the costs of different pathways, the willingness to change

paths from one setting to another, and the training required so that safety is not affected, among other factors. Patient choice should also be considered to make it easier to access the treatment needed, and to opt for different therapies with different monitoring needs. In a pandemic, like the current COVID-19 pandemic, a therapy not requiring regular blood testing of vulnerable or infected patients would be superior, but it is likely the decision would have been 
taken beforehand, without considering this nowimportant risk.

There is an appetite to transform current healthcare pathways by enhancing community services in England, ${ }^{1}$ for example, to prevent accident and emergency attendance and hospital admission of patients presenting with a possible deep vein thrombosis (DVT). In the future, suspected venous thromboembolism (VTE) management could follow similar pathways. If more were done in primary care, an improvement of patient experience as well as health outcomes would be expected. ${ }^{2}$ DVT prevalence is approximately one per $1,000^{3}$ and, consequently, is not a common presentation to general practice. Clear guidance is paramount, and evidence behind the choice of anticoagulant initiation treatment and attitudes among primary care physicians need to be considered. Variability of treatment exists as in Leeds, the CCG recommended tinzaparin in their pathway for the initial management of DVT, while less than 20 miles away, Bradford CCG recommended rivaroxaban.

According to the National Institute of Clinical Excellence (NICE), ${ }^{4}$ initial treatment should include: "an interim 24-hour dose of a parenteral anticoagulant (if a proximal leg vein ultrasound scan cannot be carried out within 4 hours)," but the advice does not include novel oral anticoagulants (NOAC), which are also indicated for DVT or VTE treatment (such as apixaban ${ }^{5}$ and rivaroxaban). ${ }^{6}$

As part of the feasibility of this study, the Cochrane library was accessed. Five Cochrane reviews with "deep vein thrombosis" and "initial treatment" in the title, abstract, or keywords were found. Three reviews focussed on pentasaccharides ${ }^{7,8}$ and non-steroidal antiinflammatories, ${ }^{9}$ which are not among the therapies suggested by NICE nor available in the pathways promoted in primary care in the UK. A fourth paper ${ }^{10}$ focussed on heparins alone, including subcutaneous unfractionated heparin (UFH), intravenous UFH, and low-molecularweight heparin $(L M W H)$, finding no difference among them. The last paper, on treatment at home versus hospital management, ${ }^{11}$ focussed on LMWH and showed no clear differences in outcomes based on location of treatment. These five works did not provide answers to the issues at hand. The aim of this review was to determine what are the best options for the initial management of DVT in primary care, considering NOAC in particular, and to combine a systematic literature review with an assessment of the current practice in England. The rationale was that a combined approach would put theory and practice together, so that more robust conclusions would be obtained.

\section{METHODS}

An initial attempt utilising the PubMed and Medline databases for keyword searches of "deep vein thrombosis," "initial treatment," and "primary care" yielded only two results, one regarding attitudes ${ }^{11}$ and the other in German. The authors then searched for the association of "deep vein thrombosis," "initial treatment," and "anticoagulant" in the NICE healthcare databases advanced search. A total of 195 articles were found among four of the databases accessed (cumulative index to nursing and allied health literature [CINAHL], EMBASE, Medline, and PubMed), although after duplications were removed 89 potential papers remained. Firstly, 19 papers were excluded as they were over 20 years old and would not adequately reflect current therapies, 18 were excluded as they were simple abstracts or posters from conferences and provided little information, seven papers were excluded due to a language barrier (three in German, two in Polish, one in Japanese, and one in Russian), and one paper was misquoted on the database and was not accessible. Finally, 21 papers were excluded as the subjects of the studies were not related to DVT drug management comparisons (Figure 1). As a result, 23 documents were obtained and further assessed, including accessing referenced papers. There were a variety of papers that provided insights for the study.

Simultaneously, freedom of information requests were sent to 199 clinical commissioning groups (CCG) in England to understand whether new pathways, like for the initial management of DVT, had been provided for general practice in the area. The standards for quality improvement reporting excellence (SQUIRE) checklist ${ }^{13}$ was the most appropriate tool available for this type of project and was used to support the manuscript structure. 


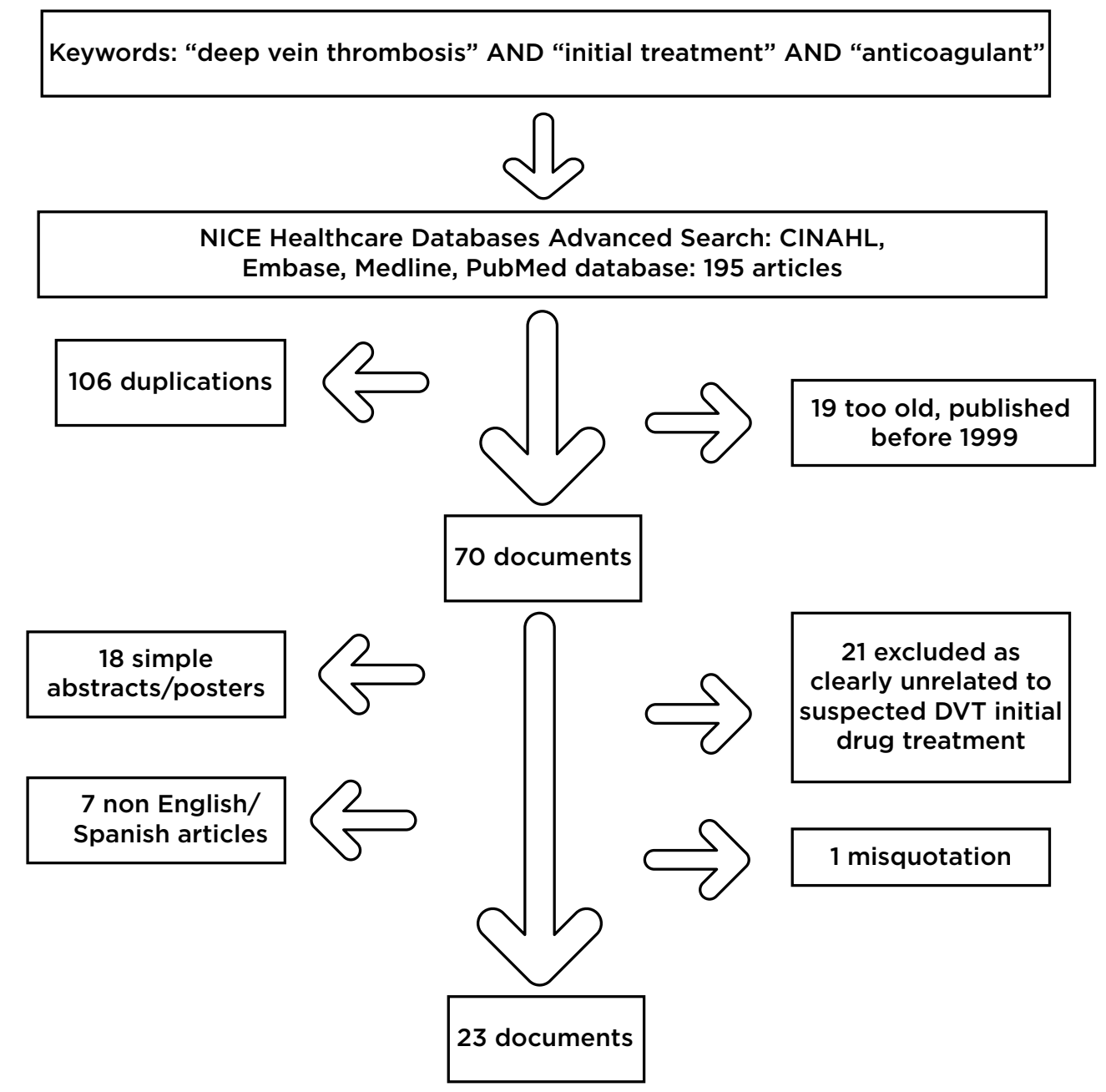

Figure 1: Flowchart of literature review paper selection process.

CINAHL: cumulative index to nursing and allied health literature.

\section{RESULTS}

The papers were studied in chronological order, looking first at long-established therapies and later at new medications available. This reflected the concept that evidence-based clinical practice is in continuous development, that guidelines need to be reviewed endlessly, and that even the way treatments are compared needs to evolve.

\section{Introduction: Long-Established Therapies}

In 2000, Douketis et al. ${ }^{14}$ focussed on factors that made recurrent VTE more likely to occur, such as the presence of cancer, chronic cardiovascular disease, chronic respiratory disease, or other clinically significant medical disease. The treatment options in that study were only UFH or a LMWH, followed by warfarin. Chong ${ }^{15}$ and Wells ${ }^{16}$ described similar management alternatives; they focussed on the possibility that home treatment with subcutaneous LMWH could be as effective as intravenous UFH. Furthermore, Harenberg ${ }^{17}$ concluded that LMWH was superior to intravenous heparin, and Hull and Pineo ${ }^{18}$ concluded that UFH should no longer be standard therapy, replaced by LMWH. NOAC were not part of the available therapies at the time.

Huisman and Bounameaux, ${ }^{19}$ as well as Schulmann, ${ }^{20}$ assessed the benefits of LMWH and considered the benefits of a new oral medication, ximelagatran, but this medication was later withdrawn due to hepatic toxicity. ${ }^{21} \mathrm{~A}$ systematic review by $\mathrm{Akl}$ et al. $^{22}$ considered 
that among patients with cancer, LMWH was probably superior to UFH. Initial anticoagulation for patients with confirmed or highly suspected DVT, according to the American College of Chest Physicians evidence-based clinical practice guidelines from 2008, ${ }^{21}$ included shortterm treatment with subcutaneous LMWH, intravenous UFH, monitored subcutaneous UFH, fixed-dose subcutaneous UFH, or subcutaneous fondaparinux. There was no preference indicated. Not long after, it was accepted that LMWH had taken over from UFH as the initial treatment of choice. ${ }^{23,24}$

\section{Novel Oral Anticoagulants}

In 2010, NOAC were undergoing trials and expected to soon be in the market. ${ }^{24}$ They included dabigatran, targeting thrombin (factor Ila), and apixaban and rivaroxaban, targeting factor Xa. By 2013 there were limited data about NOAC to allow for recommendation of their use to treat VTE in patients with cancer. ${ }^{25}$ Rivaroxaban, apixaban, edoxaban, and dabigatran were considered to have similar efficacy and safety to conventional standard-of-care anticoagulation. Because of this, according to McRae, ${ }^{26}$ clinical judgement regarding the optimal choice of anticoagulant therapy for VTE should have been based on individual patient characteristics. NOAC were not commonly used, and the main reasons preventing their use were costeffectiveness and lack of a reversal agent. ${ }^{12}$ Interestingly, Maervoet et al. ${ }^{27}$ considered rivaroxaban a cost-effective therapy.

With improved safety and effectiveness of medications like rivaroxaban ${ }^{28}$ and edoxaban ${ }^{29}$ compared to standard anticoagulants, NOAC were positioning themselves where LMHW had in comparison to UFH eight years earlier, and physicians conservatively increased their use of NOAC instead of LMWH. ${ }^{30}$ In Europe, the initial VTE treatment was parenteral heparin in approximately $66 \%$ of cases, while NOAC monotherapy prescriptions represented approximately $19 \%{ }^{31}$

Another issue reflected in the literature was the switching of anticoagulant treatment once initiated as, for example, one analysis noted that among patients with cancer: "Approximately one-quarter of patients who initiated on LMWH switched to other anticoagulant agents during the course of treatment compared to $8 \%$ and $5 \%$ of patients observed with warfarin and rivaroxaban respectively."32 Turple et al. ${ }^{33}$ suggested that physicians prescribe standard anticoagulation to many higher-risk patients before starting rivaroxaban. It was also noted that switching to rivaroxaban did not appear to adversely affect treatment outcomes, despite the greater presence of risk factors in those who were switched early. ${ }^{33}$

Although NOAC were not commonly prescribed in a paper by Mausbach et al., ${ }^{34}$ it was concluded that ambulatory treatment was safe. Guidelines were changing and NOAC were considered the first-line therapeutic agent instead of warfarin. ${ }^{35}$ There were still specific groups of patients where LMWH remained the anticoagulant of choice, namely pregnant women and patients who are obese. $^{36}$

\section{Pathways in Practice}

Several CCG in England have pathways that allow patients to start anticoagulation in primary care while waiting for confirmation of the diagnosis in secondary care. Using freedom of information requests to CCG, the number of CCG implementing community services for the management of DVT was identified, and the variability of approaches regarding the choice of anticoagulation noted. Among the 177 CCG that responded, only a few had pathways that involved general practitioners prescribing the initial anticoagulation. Three CCG had an injectable therapy as the initial anticoagulation: enoxaparin in Ipswich and East Suffolk CCG, and tinzaparin in Leeds CCG and Telford and Wrekin CCG. NOAC were the recommended initial therapy in the pathways of 11 CCG: apixaban in Brighton CCG and Sunderland CCG; apixaban or rivaroxaban in Eastern Cheshire CCG, Hambleton, Richmond, and Whitby CCG, and Northumberland CCG; rivaroxaban in Bradford CCG, Greater Huddersfield CCG, Harrogate CCG, Kernow CCG, and Wiltshire CCG; and a non-specified NOAC in Portsmouth CCG. One CCG pathway provided the option to choose an injectable or NOAC therapy, in County Durham and Darlington CCG.

Globally, NOAC have been widely used with or without a parenteral anticoagulant lead-in, but this varies geographically; ${ }^{37}$ recent studies showed that NOAC were given to approximately 
$60 \%$ of patients on anticoagulants in Europe and Asia, but were given to $<30 \%$ in Latin America and the Middle East. ${ }^{38}$ Use of NOAC also depended on subpopulations of patients, ${ }^{37}$ as they were less frequently used among patients who had cancer, chronic renal disease, heart failure, or prior stroke, ${ }^{38}$ despite the efficacy and safety of NOAC being similar to, ${ }^{39}$ or better than, ${ }^{40}$ LMWH in patients with cancer, for example. NOAC use in specific populations remains challenging, such as in Asian populations or patients with cancer. ${ }^{41}$

\section{DISCUSSION}

This study combined an extensive literature review with freedom of information requests on current practice in England. This unique approach provided an understanding of which evidencebased VTE management is practised, and specifically which initial treatment of DVT is used in primary care in the UK.

\section{Summary}

Initial management of DVT no longer needs to be hospital based. New developments in pharmacology with LMWH and NOAC have allowed general practice to step in and offer patients a service that is more convenient. Looking at the literature available, it is clear there has been a progression of the options available for the management of VTE, and there is noninferiority between LMWH and NOAC. However, the updated Chest guidelines ${ }^{42}$ make a distinction for choice of therapy for patient subgroups: for patients without cancer, dabigatran, rivaroxaban, apixaban, or edoxaban are recommended, while if the patient has cancer-associated thrombosis, the first-line therapy is LMWH. Also of note is that initial parenteral anticoagulation should be given before dabigatran and edoxaban, but not before rivaroxaban and apixaban. ${ }^{42}$

General practitioners in the UK follow pathways designed locally, and in this study CCG approach was variable. Only one of the 15 CCG offering primary care DVT pathways allowed the possibility for choosing injectable therapies or NOAC, while the others were more restrictive. Considering that there is no inferiority among the different options currently available, these pathways could better accommodate patient choice.
Deciding between oral and injectable therapies is likely important to patients, but it was considered by only one of the CCG. Benefits of NOAC, including predictable dose response, lack of need for monitoring, reduced need for drug adjustment, absence of food interactions, and limited drug interactions, ${ }^{43}$ would probably attract more patients compared to short-term injectable therapies with a probable switch to warfarin. In the long run, quality of life was comparable between NOAC and warfarin therapies but NOAC treatment resulted in higher treatment satisfaction; ${ }^{44}$ the fact that regular monitoring is required with warfarin was an issue probably not fully considered. Discussion of monitoring requirements with patients should include risks associated with mobility and travel changes, because of shielding or self-isolating in a pandemic like the current COVID-19 pandemic, change of address to more remote places, or even holiday plans.

Clinicians have different concerns to their patients. Issues including the need for patient weight to calculate the dose of LMWH, limited experience with this diagnosis or with prescribing adequate doses of NOAC, and the time requirements for the treatment puts additional pressure on general practitioners. A recent survey in a hospital setting in Qatar found that confidence in prescribing NOAC was very limited $^{45}$ which should raise concerns when new pathways are implemented. Othieno et al. ${ }^{11}$ showed that LMWH treatment in the community was no worse than hospital treatment, but there is a need to explore further as NOAC are now more commonly used. Furthermore, NOAC are not always prescribed appropriately ${ }^{46}$ and attitudes, although explored elsewhere, ${ }^{12,45}$ are not necessarily progressing. For clinicians, management plan decisions could also be affected by whether the patient is affected by a high-risk infection like COVID-19 or if the services are limited because of other factors.

The situation is still evolving as the systematic review recently published by Wang et al., ${ }^{47}$ in opposition to the recent Chest guidelines, ${ }^{36}$ concluded that NOAC were the first choice for treatment in patients with cancer. NOAC are increasingly replacing previous agents as first choice for the management of VTE. 


\section{Strengths and Limitations}

The combined approach, assessing what is happening in general practice in England and what are the recommendations on treatment, provided a clear understanding of initial management of DVT in England, showing its variability. However, there is little use of community pathways for the initial treatment of DVT, with many areas heavily dependent on secondary care. Treatment options vary but there is no clear more efficient therapy, nor indication that primary care pathways are better. Confidence and safe prescribing are issues highlighted, and in need of further analysis.

\section{CONCLUSIONS}

Primary care is starting to manage DVT initial prescription and to organise tests without the need for patients to attend accident and emergency, as was previous practice. Policies are variable, as are clinicians' expertise and patient options. Implementation of new guidelines in general practice should be more uniform and less dependent on geography, and patients should be given more choice, which would then allow research to produce clearer guidance.

\section{Implications for Research and Practice}

There are still knowledge gaps regarding the safety of initial treatment of DVT in the community, as clinicians' choices are based on what is available in their CCG and the expertise on the matter is limited. General practitioners' confidence in prescribing NOAC needs further assessment.

\section{References}

1. Department of Health. Our health, our care, our say: a new direction for community services. 2006. Available at: https://assets.publishing. service.gov.uk/government/uploads/ system/uploads/attachment_data/ file/272238/6737.pdf. Last accessed: 29 May 2020.

2. All-Party Parliamentary Thrombosis Group. NHS innovation showcase: DVT diagnosis and treatment in Primary care. 2015. Available at: http://apptg.org.uk/wp-content/ uploads/2016/12/NHS-InnovationShowcase.pdf. Last accessed: 01 June 2020

3. Keeling DM et al. The diagnosis of deep vein thrombosis in symptomatic outpatients and the potential for clinical assessment and D-dimer assays to reduce the need for diagnostic imaging. $\mathrm{Br} \mathrm{J}$ Haematol. 2004;124(1):15-25.

4. National Institute for Health and Care Excellence (NICE). CG144: Venous thromboembolic diseases: diagnosis, management and thrombophilia testing. 2012. Available at:www. nice.org.uk/guidance/cg144/ resources/venous-thromboembolicdiseases-diagnosis-managementand-thrombophilia-testingpdf-35109570835141. Last accessed: 11 August 2019

5. National Institute for Health and Clinical Excellence (NICE). TA341: Apixaban for the treatment and secondary prevention of deep vein thrombosis and/or pulmonary embolism. 2015. Available at: www. nice.org.uk/guidance/ta341. Last accessed: 11 August 2019.

6. National Institute for Health and Clinical Excellence (NICE). TA261: Rivaroxaban for the treatment of deep vein thrombosis and prevention of recurrent deep vein thrombosis and pulmonary embolism. 2012. Available at: www.nice.org.uk/ guidance/ta261. Last accessed: 11 August 2019.

7. Brandao GMS et al. Pentasaccharides for the treatment of deep vein thrombosis. Cochrane Database Syst Rev. 2017;12:CD011782. doi:10.1002/14651858.CD011782.pub2.

8. Hakoum MB et al. Anticoagulation for the initial treatment of venous thromboembolism in people with cancer. Cochrane Database Syst Rev. 2018;1(1):CD006649. doi:10.1002/14651858.CD006649. pub7.

9. Cundiff DK et al. Anticoagulants versus non-steroidal antiinflammatories or placebo for treatment of venous thromboembolism. Cochrane Database Syst Rev. 2006;1:CD003746. doi:10.1002/14651858.CD003746. pub2.

10. Robertson L, Strachan J. Subcutaneous unfractionated heparin for the initial treatment of venous thromboembolism. Cochrane Database Syst Rev. 2017;2:CD006771. doi:10.1002/14651858.CD006771.pub3.

11. Othieno R et al. Home versus inpatient treatment for deep vein thrombosis. Cochrane Database Syst Rev. 2018;1:CD003076. doi:10.1002/14651858.CD003076. pub3.

12. Connell NT, Butera JN. Attending physician attitudes toward choice of oral anticoagulant for the treatment of venous thromboembolism. R I Med J. 2015;98(7):32-6.

13. Ogrinc $\mathrm{G}$ et al. SQUIRE 2.0 (Standards for QUality Improvement Reporting Excellence): revised publication guidelines from a detailed consensus process. BMJ Qual Saf, 2016;25(12):986-992.

14. Douketis JD et al. Clinical risk factors and timing of recurrent venous thromboembolism during the initial 3 months of anticoagulant therapy. Arch Intern Med. 2000;160(22):34316.

15. Chong $\mathrm{BH}$. Management of deep vein thrombosis. Aust Fam Physician. 2001;30(3):235-40.

16. Wells PS. Outpatient treatment of patients with deep-vein thrombosis or pulmonary embolism. Curr Opin Pulm Med. 2001;7(5):360-4.

17. Harenberg J. Fixed-dose versus adjusted-dose low molecular weight 
heparin for the initial treatment of patients with deep venous thrombosis. Curr Opin Pulm Med. 2002;8(5):383-8.

18. Hull RD, Pineo GF. Heparin and lowmolecular-weight heparin therapy for venous thromboembolism: will unfractionated heparin survive? Semin Thromb Hemost. 2004;30(Suppl 1):11-23.

19. Huisman MV, Bounameaux $\mathrm{H}$. Treating patients with venous thromboembolism: initial strategies and long-term secondary prevention. Semin Vasc Med. 2005;5(3):276-84.

20. Schulman S. The Role of Ximelagatran in the Treatment of Venous Thromboembolism. Pathophysiol Haemost Thromb. 2005;34(1):18-24.

21. Kearon $\mathrm{C}$ et al. Antithrombotic therapy for venous thromboembolic disease: American College of Chest Physicians evidence-based clinical practice guidelines (8th Edition). Chest. 2008;133(6 Suppl):454S-545S

22. Akl EA at al. Anticoagulation in patients with cancer: an overview of reviews. Pol Arch Intern Med. 2008;118(4):183-93.

23. Mainwaring $\mathrm{C}$, Bhandari S. Diagnosis and management of deep vein thrombosis. Acute Med. 2009;8(2):53-62.

24. Ageno W. Recent advances in the management of venous thromboembolism. Korean J Hematol. 2010;45(1):8-13.

25. Gómez-Outes A et al. Potentia role of new anticoagulants for prevention and treatment of venous thromboembolism in cancer patients. Vasc Health Risk Manag. 2013;9:20728.

26. Mcrae S. Treatment options for venous thromboembolism: lessons learnt from clinical trials. Thromb J. 2014;12(1):27.

27. Maervoet $\mathrm{J}$ et al. Cost effectiveness of rivaroxaban versus low molecular weight heparin and vitamin $\mathrm{K}$ antagonists for the treatment of deep-vein thrombosis in the Belgian healthcare setting. Eur J Cardiovasc Med. 2015;3(1):452-61.

28. Ageno $W$ et al. Safety and effectiveness of oral rivaroxaban versus standard anticoagulation for the treatment of symptomatic deep-vein thrombosis (XALIA): An international prospective non-interventional study. Lancet Haematol. 2016; 3(1):e12-21.

29. Brekelmans MPA et al. Clinical impact and course of major bleeding with edoxaban versus vitamin $\mathrm{K}$ antagonists. Thromb Haemost. 2016;116(1):155-61.

30. Dault R et al. Patterns and predictors of use of anticoagulants for the treatment of venous thromboembolism following approval of rivaroxaban. Clin Appl Thromb Hemost. 2016;22(8):765-71.

31. Guercini $\mathrm{F}$ et al. The management of patients with venous thromboembolism in Italy: insights from the PREFER in VTE registry. Intern Emerg Med. 2016;11(8):1095102.

32. Khorana AA et al. Current practice patterns and patient persistence with anticoagulant treatments for cancerassociated thrombosis. Res Pract Thromb Haemost. 2017;1(1):14-22.

33. Turpie AGG et al. Analysis of patients with deep vein thrombosis switched from standard therapy to rivaroxaban in the non-interventional XALIA study. Thromb Res. 2017;155:23-7.

34. Mausbach LS et al Ambulatory versus in-hospital treatment of proximal lower-limb deep vein thrombosis in adults: a retrospective cohort study. Clin Appl Thromb Hemost. 2017:23(7):859-64.

35. Okumura $Y$ et al. Design and rationale for the Japanese registry of rivaroxaban effectiveness \& safety for the prevention of recurrence in patients with deep vein thrombosis and pulmonary embolism (J'xactly) study. BMJ Open. 2018;8(6):e020286.

36. Brenner B et al. Evaluation of unmet clinical needs in prophylaxis and treatment of venous thromboembolism in at-risk patient groups: pregnancy, elderly and obese patients. Thromb J. 2019:17(1):6.

37. Haas S et al. Management of venous thromboembolism in routine clinical practice - results from the global GARFIELDVTE registry. Hamostaseologie. 2019:39(Suppl1):S1-92
38. Goldhaber SZ et al. Profile of patients diagnosed with acute venous thromboembolism in routine clinical practice: the RE-COVERY DVT/PE ${ }^{\mathrm{TM}}$ study. Am J Med. 2020. doi:10.1016/j. amjmed.2020.03.036.

39. Stepien $\mathrm{K}$ et al. Extended treatment with non-vitamin $\mathrm{K}$ antagonist oral anticoagulants versus low-molecularweight heparins in cancer patients following venous thromboembolism. A pilot study. Vascul Pharmacol. 2019;120:106567.

40. Wu VCC et al. Novel oral anticoagulant versus warfarin in cancer patients with atrial fibrillation: an 8-year population-based cohort study. J Cancer. 2020;11(1):92-9.

41. Cho IY. Using non-vitamin K oral anticoagulants in specific patient populations: a study of Korean cases. Ther Clin Risk Manag. 2019;15:1183206.

42. Kearon $\mathrm{C}$ et al. Antithrombotic therapy for VTE Disease: CHEST guideline and expert panel report. Chest. 2016;149(2):315-52.

43. Baglin T. Clinical use of new oral anticoagulant drugs: dabigatran and rivaroxaban. $\mathrm{Br} \mathrm{J}$ Haematol 2013;163:160-7.

44. Ng DLC et al. Comparing quality of life and treatment satisfaction between patients on warfarin and direct oral anticoagulants: a crosssectional study. Patient Prefer Adherence. 2019;13:1363-73.

45. El-Bardissy A et al. A survey on the awareness and attitude of physicians on direct oral anticoagulants in Qatar. Clin Appl Thromb Hemost. 2018;24(9 Suppl):255S-60S.

46. Elewa $\mathrm{H}$ et al. Appropriateness of dabigatran and rivaroxaban prescribing in Qatar: a 5-year experience. J Cardiovasc Pharmacol Ther. 2018;23(2):155-61.

47. Wang $Y$ et al. Efficacy and safety of direct oral anticoagulants for secondary prevention of cancerassociated thrombosis: a systematic review and meta-analysis of randomized controlled trials and prospective cohort studies. Front Pharmacol, 2019;10:773. 